
$\underbrace{6}_{\text {JगPBA }}$
Contents lists available at www.ijpba.in
International Journal of Pharmaceutical and Biological Science Archive
NLM (National Library of Medicine ID: 101738825)
Index Copernicus Value 2019: 71.05
Volume 9 Issue 2; March-April; 2021; Page No. 24-30

\title{
OVERVIEW OF AN OVARIAN CANCER AND ITS TREATMENT ASPECTS
}

\author{
Vaghasiya Tushar Kishorbhai ${ }^{1}$, Miteshkumar Malaviya ${ }^{2^{*}}$ \\ ${ }^{1,2}$ Shree Dhanvantry Pharmacy College, Gujarat, India \\ Conflicts of Interest: Nil \\ Corresponding author: Miteshkumar Malaviya \\ DOI: https://doi.org/10.32553/ijpba.v9i2.185
}

\begin{abstract}
Cancer (medical term: malignant neoplasm) is a class of diseases in which a group of cells display uncontrolled growth, invasion that intrudes upon and destroys adjacent tissues, and sometimes metastasis, or spreading to other locations in the body via lymph or blood. These three malignant properties of cancers differentiate them from benign tumors, which do not invade or metastasize. Ovarian cancer is fundamentally a disease of failure of regulation of tissue growth. In order for a normal cell to transform into a cancer cell, the genes which regulate cell growth and differentiation must be altered. These brief surveys discuss the current therapy, novel treatment and its side effects.
\end{abstract}

Keywords: Cancer, Cancer treatment, Liposomal formulation

\section{Introduction}

The body is made up of trillions of living cells. Normal body cells grow, divide, and die in an orderly way. During the early years of a person's life, normal cells divide faster to allow the person to grow. After the person becomes an adult, most cells divide only to replace wornout, damaged, or dying cells. Cancer begins when cells in a part of the body start to grow out of control. There are many kinds of cancer, but they all start because of this out-of-control growth of abnormal cells. Cancer cell growth is different from normal cell growth. Instead of dying, cancer cells keep on growing and form new cancer cells. These cancer cells can grow into (invade) other tissues, something that normal cells cannot do. Being able to grow out of control and invade other tissues is what makes a cell a cancer cell. When cancer cells get into the bloodstream or lymph vessels, they can travel to other parts of the body. There they begin to grow and form new tumors that replace normal tissue. This process is called metastasis. Not all tumors are cancerous. Tumors that aren't cancer are called benign. Benign tumors can cause problems they can grow very large and press on healthy organs and tissues. But they cannot grow into other tissues. Because of this, they also can't spread to other parts of the body (metastasize). These tumors are almost never life threatening (1).

Ovarian cancer is the fifth leading cause of death from cancer in women and the leading cause of death from gynecological cancer, resulting in approximately 21,880 estimated new cases in 2012 with an estimated 13,850 deaths in 2010 in the USA (2), (3). Epithelial ovarian cancer arising from the ovarian surface epithelium (OSE) account for $\sim 90 \%$ of ovarian malignancies. Clinically, epithelial ovarian cancer often involves the ovary and omentum, with diffuse, multi-focal intraperitoneal metastases and malignant ascites (4). Although the lifetime risk of developing ovarian cancer is relatively low (1.4\% to $1.8 \%)(5)$, the mortality rate for this disease is high. Currently, there is no effective method of screening for early stage ovarian cancer in the general population. Moreover, typical symptoms in affected women are nonspecific and of a gastrointestinal or abdominopelvic nature, which commonly delays a confirmed diagnosis (6), (7). As a result, most women $(70 \%$ to $75 \%)$ have advanced-stage (III-IV) disease at the time of presentation (8), (9). 


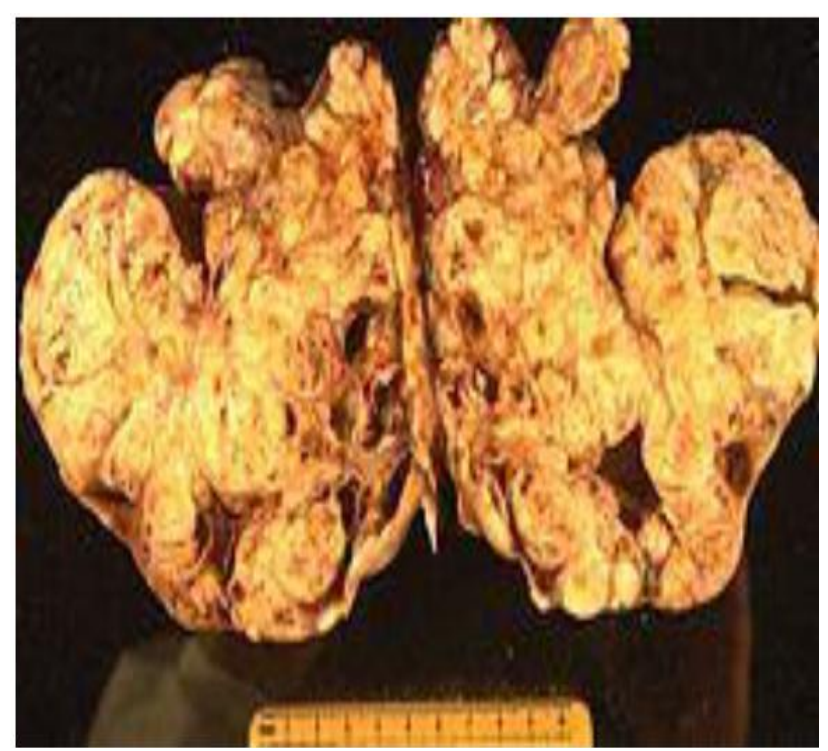

Figure 1.1: A pathological specimen of ovarian carcinoma

For treatment purpose, after initial surgery, the combination of platinum (cisplatin and carboplatin) plus taxanes (paclitaxel and docetaxel) represents the standard first line treatment in advanced ovarian cancer (10), (11). Despite a high initial response, the patients often relapse within a median time of less than 2 years (12). The current chemotherapy is associated with serious side effects such as neutropenia and neurotoxicity due to the nonspecific biodistribution and low therapeutic index of anticancer agents (13). New drug formulations and targeting approaches have been developed to better control the biodistribution of drugs and to improve the therapeutic efficacy (14).

Treatment cycle of combination therapy of paclitaxel and carboplatin for ovarian cancer:

6 cycles of paclitaxel plus carboplatin is given on day 1 of 21 day cycle ( 3 weeks $\times 6$ cycles). Carboplatin at AUC of 5-7.5 i.v. infusion for an hour on day 1 , with paclitaxel at dose of 175 $\mathrm{mg} / \mathrm{m} 2$ over 3 hour infusion on day 1 .

Paclitaxel is a novel antimicrotubule agent that promotes the assembly of microtubules from tubulin dimers and stabilizes microtubules by preventing depolymerization. This stability inhibits the normal dynamic reorganization of the microtubule network that is essential for vital interphase and mitotic cellular functions.
In addition, paclitaxel induces abnormal arrays or bundles of microtubules throughout the cell cycle and multiple asters of microtubules during mitosis, further disrupting cell function (15).

The current clinical dosage form of paclitaxel (Taxol®), which is parenteral, is dissolved in a mixture of Cremophor ${ }^{\circledR}$ EL (poly-oxyethylated caster oil) and ethanol (50:50, v/v) and needs to be diluted right before injection. However, Cremophor® EL has been associated with serious side-effects and leads to hypersensitivity, nephrotoxicity and neurotoxicity in many patients (S) (16). Although a premedication regimen with corticosteroids and antihistamine reduces the incidence of serious hypersensitivity, milder reactions have still occurred in $5-30 \%$ of patients (17), (18).

Carboplatin is an antineoplastic in the class of alkylating agents and is used to treat various forms of cancer. Carboplatin, a cisplatin analog, is an antineoplastic drug with an activity profile similar to cisplatin. However, the toxic effects of carboplatin include dose related myelosupression with severe thrombocytopenia and leucopenia, especially in older patients with renal impairment or who have previously received chemotherapy (19). The main side effects of carboplatin were its toxicity to the kidney and the damage to marrow, with severe thrombocytopenia and leucopenia. Carboplatin (Paraplatin) is available as aqueous solution.

One strategy that can be used to overcome the side effects and occurrence of resistance to platinum drugs; and to increase its bioavailability and therapeutic efficacy is to encapsulate them in liposomes.

\section{Problems related to current standard treatment of ovarian cancer:}

\section{Pharmaceutical Aspects:}

Paclitaxel is poorly soluble in an aqueous medium so, Paclitaxel is currently formulated (Taxol®) in a vehicle composed of 1:1 blend of Cremophor EL (polyethoxylated castor oil) and ethanol which is diluted with 5-20-fold in normal saline or dextrose solution $(5 \%)$ for administration. This formulation is stable in unopened vials for 5 years at $4{ }^{\circ} \mathrm{C}$. However, 
lots of problems employing this vehicle have been reported:

\section{Vehicle related problems}

One of the substantial problems associated with this formulation is that the ethanol: Cremophor vehicle required to solubilize it, is toxic (20). Although it has been used to administer other drugs such as cyclosporine (21) and teniposide1, the amount of Cremophor necessary to deliver the required doses of paclitaxel is significantly higher than that of administered with any other marketed drug (22). The side effects caused by Cremophor EL include hypersensitivity reactions, nephrotoxicity and neurotoxicity. Cremophor EL also has an influence on the function of endothelial and vesicular muscles and causes vasodilation, labored breathing, lethargy and hypotension (23), (24).

Premedication with corticosteroids (dexamethasone) and antihistamine (both $\mathrm{H} 1$ and H2-receptor antagonist) is used with paclitaxel to increase safety and reduce intensity and the incidence of serious hypersensitivity reactions associated with paclitaxel administration in Cremophor (25). The doses of dexamethasone given are relatively large, and certain H2-receptor antagonist such as cimetidine and ranitidine is well known inhibitors of the cytochrome P-450 metabolic pathway (26) and the later is thought to be involved in the metabolism of paclitaxel (27)suggesting the potential for interaction.

(2) Paclitaxel/ethanol: Cremophor formulation also shows an incompatibility with the components of the infusion sets. It was reported (28), (29), (30) that both ethanol and Cremophor leach diethylhexylpthalate (DHEP) from the polyvinylchloride (PVC) infusion bag and administration sets.

(3) The use of Cremophor as a vehicle also appears to alter the biochemical properties of lipoproteins, such as high-density lipoproteins. It has also been shown to partially mediate the cytotoxic activity of paclitaxel in primary cultures of tumor cells from patients and to reverse P-glycoprotein mediated multidrug resistance (31), (32).

\section{Formulation related problems}

The recommended concentration of the drug in the properly diluted clinical formulation is 0.3 $1.2 \mathrm{mg} \mathrm{ml}-1$ (0.35-14 mM) and has only shortterm physical stability as some particles slowly tend to precipitate out of the aqueous media. The stability of appropriately diluted paclitaxel was estimated at $12-24 \mathrm{~h}$ and its use was recommended within $12 \mathrm{~h}$ of dilution in the aqueous media. It is suggested that drug is administered promptly after dilution.

No pharmaceutical problems related to marketed formulation of Carboplatin. (Paraplatin, injection).

\section{Pharmacological aspects:}

Paclitaxel: Low therapeutic Index, All common side effects related to chemotherapeutic drugs, very less solublity in water $(1 \mu \mathrm{g} / \mathrm{ml})$.

Carboplatin: Dose related myelosupression with severe thrombocytopenia and leucopenia most prominent, nephrotoxicity, Neurotoxocity.

\section{Liposome}

If drugs having low therapeutic indices could be delivered in higher concentrations to their target cells (thereby increasing efficacy) and away from normal cells (thereby reducing toxicity), this would provide us with means of increasing the therapeutic indices of the drugs, achieving more effective therapy and possibly greater economic benefit (33). An alternative approach consists in associating the drug to a submicroscopic carrier thereby hiding and protecting the molecule from degradation and delivering it to inaccessible target cells in a controlled manner. More recently liposome drug delivery systems are being utilized in an effort to reduce the dose-associated toxicity while enhancing efficacy. The pioneer concept of targeted drugs was postulated by Erlich in 1906 (34)and defined as the magic bullet, but as stressed by R. Duncan (1997), the drug development process is inevitably lengthy and breakthroughs more frequently a dream rather than reality (35).

Liposomes can be used as drug carriers, entrapping drugs in the bilayer core or in the outer domain. Designed as carriers of anticancer 
drugs, liposomes exhibit affinity for tumor cells and target the lymph system, changing the distribution of drugs in tissues and selectively killing tumor cells or restraining their reproduction. In this way, they can improve the therapeutic efficacy and reduce toxicity, as well as metamorphic reactions and immunoreactions. Liposomes can be formulated from a variety of lipid and lipid mixtures with different compositions and can be modified in terms of their particle size, structure and surface charge for the purpose of obtaining desirable physicochemical properties to suit particular needs (36).

In spite of the obvious advantages, development of liposomal formulation poses several challenges. First, intravenously administered liposomes are taken up by the cells of mononuclear phagocytic system (MPS) in dozens of minutes. Even faster, they can lose the encapsulated drugs, especially drugs with a low value of water/octanol partition coefficient. It was proposed that the rapid clearance of liposomes is due to the uptake by the cells of the body"s immune system as well as disintegration upon interactions with plasma lipoproteins. Proteins of the immune system adsorb onto liposomes immediately after injection and this opsonization tags them for subsequent macrophage uptake. The molecular origin of these interactions are mostly long range electrostatic, Van-der Waals and short range hydrophobic interactions of liposomes with macromolecules in the blood. While electrostatic and hydrophobic interactions can be minimized by using neutral lipids and mechanically very strong bilayers, Van-der Waals attraction is ubiquitous. However, it shows exponential decay with distance. Van-der Waals attraction can be, therefore, reduced by coating the liposome surface with inert polymers which prevent the close approach of proteins. This process is sometimes utilized by macrophage - mediated delivery system (37), (38).

The second problem of liposomes is rapid leakage of encapsulated drug. Typically, halflives for passively encapsulated drug range from minutes in Egg PC bilayers to a dozen hours in the case of mechanically strongest bilayers, such as DSPC/ CHOL. Efficiency of loading can be greatly increased by using a $\mathrm{pH}$ gradient loading technique.

Generally speaking, phagocytes are detrimental to liposomes and engulf them within minutes. Camouflaging the liposomes so as to avoid engulfment by phagocytes thus became a key objective of pharmaceutical chemists for a very long time. The result of their efforts was a process called PEGylation (39), in which countless molecules of a synthetic, non - toxic polymer, polyethylene glycol (PEG), are attached, at one end of the polymer chain, to the surface of the liposome. Because of their chemical affinity for water molecules, they are heavily hydrated. To phagocytes, this molecular "cloak" of water of hydration makes the PEGylated liposomes look like little watery blobs rather than something edible, so they tend to leave them alone. The two main advantages of PEGylated liposomes for delivering drugs or supplements are increased bioavailability (40).

and the possibility, in some cases, of targeted delivery to the organs or tissues that most need them.

There are various advantages of liposomes. Liposomes increase bioavailability and provide targeted delivery in some cases. They also keep the maximum concentration of drug lower than conventional formulations at all times by providing slow and constant release of liposomes. Pegylation provides additional advantage of by passing the reticuloendothelial system (RES) and preventing drug leakage. Also it provides even more delayed release as the PEGylated chain takes a long time to breakdown.

\section{Conclusion}

Liposomes of both the drugs were prepared because of carboplatin liposomal formulation was prepared to overcome the side effects and occurrence of resistance to platinum drugs; and to increase its bioavailability and therapeutic efficacy. Paclitaxel liposomal formulation was prepared to develop aqueous based formulation without use of cremophor EL and so as to avoid serious side effects related to this vehicle and to increase the therapeutic efficiency Overcome 
pharmaceutical problems related to marketed formulation of paclitaxel (Taxol) i.e. eliminate toxicities associated with cremophor and ethanol. Decreased RES uptake and prolonged circulation time. Passive targeting to tumor tissues and control bio-distribution of drugs. Increased efficacy and therapeutic index. Reduction in toxicity of encapsulated agents: to eliminate serious dose related myelosupression with severe thrombocytopenia and leucopenia with use of carboplatin; systemic toxicities of paclitaxel.

\section{References}

1. Croce CM. Oncogenes and cancer. The New England journal of medicine. 2008;358(5):502-11.

2. Kim PS, Djazayeri S, Zeineldin R. Novel nanotechnology approaches to diagnosis and therapy of ovarian cancer. Gynecologic oncology. 2011;120(3):393403.

3. Jemal A, Siegel R, Xu J, Ward E. Cancer statistics, 2010. CA: a cancer journal for clinicians. 2010;60(5):277-300.

4. Selvaggi SM. Tumors of the Ovary, Maldeveloped Gonads, Fallopian Tube, and Broad Ligament. Archives of Pathology \& Laboratory Medicine. 2000;124(3):474-.

5. Cristea M, Han E, Salmon L, Morgan RJ. Practical considerations in ovarian cancer chemotherapy. Ther Adv Med Oncol. 2010;2(3):175-87.

6. Memarzadeh S, Berek JS. Advances in the management of epithelial ovarian cancer. The Journal of reproductive medicine. 2001;46(7):621-9; discussion 930.

7. Bhatt P, Vhora I, Patil S, Amrutiya J, Bhattacharya C, Misra A, et al. Role of antibodies in diagnosis and treatment of ovarian cancer: Basic approach and clinical status. Journal of Controlled Release. 2016;226:148-67.

8. Partridge EE, Phillips JL, Menck HR. The National Cancer Data Base report on ovarian cancer treatment in United States hospitals. Cancer. 1996;78(10):2236-46.
9. Goff BA, Mandel L, Muntz HG, Melancon CH. Ovarian carcinoma diagnosis. Cancer. 2000;89(10):2068-75.

10. Cirstoiu-Hapca A, Buchegger F, Lange N, Bossy L, Gurny R, Delie F. Benefit of anti-HER2-coated paclitaxel-loaded immuno-nanoparticles in the treatment of disseminated ovarian cancer: Therapeutic efficacy and biodistribution in mice. Journal of controlled release : official journal of the Controlled Release Society. 2010;144(3):324-31.

11. Pfisterer J, du Bois A, Wagner U, Quaas $\mathrm{J}$, Blohmer JU, Wallwiener $\mathrm{D}$, et al. Docetaxel and carboplatin as first-line chemotherapy in patients with advanced gynecological tumors. A phase I/II trial of the Arbeitsgemeinschaft Gynäkologische Onkologie (AGO-OVAR) Ovarian Cancer Study Group. Gynecologic oncology. 2004;92(3):949-56.

12. Muggia FM, Braly PS, Brady MF, Sutton G, Niemann TH, Lentz SL, et al. Phase III randomized study of cisplatin versus paclitaxel versus cisplatin and paclitaxel in patients with suboptimal stage III or IV ovarian cancer: a gynecologic oncology group study. Journal of clinical oncology : official journal of the American Society of Clinical Oncology. 2000;18(1):106-15.

13. McGuire WP, 3rd, Markman M. Primary ovarian cancer chemotherapy: current standards of care. Br J Cancer. 2003;89 Suppl 3(Suppl 3):S3-S8.

14. Du Bois A, Pfisterer J. Future options for first-line therapy of advanced ovarian cancer. International journal of gynecological cancer : official journal of the International Gynecological Cancer Society. 2005;15 Suppl 1:42-50.

15. Vhora I, Patil S, Bhatt P, Misra A. Chapter One - Protein- and Peptide-Drug Conjugates: An Emerging Drug Delivery Technology. In: Donev R, editor. Advances in Protein Chemistry and Structural Biology. 2015;98:1-55.

16. Singla AK, Garg A, Aggarwal D. Paclitaxel and its formulations. International journal of pharmaceutics. 2002;235(1-2):179-92. 
17. Weiss RB, Donehower RC, Wiernik PH, Ohnuma T, Gralla RJ, Trump DL, et al. Hypersensitivity reactions from taxol. Journal of clinical oncology : official journal of the American Society of Clinical Oncology. 1990;8(7):1263-8.

18. Yewale C, Baradia D, Patil S, Bhatt P, Amrutiya J, Gandhi R, et al. Docetaxel loaded immunonanoparticles delivery in EGFR overexpressed breast carcinoma cells. Journal of Drug Delivery Science and Technology. 2018;45:334-45.

19. Wagstaff AJ, Ward A, Benfield P, Heel RC. Carboplatin. A preliminary review of its pharmacodynamic and pharmacokinetic properties and therapeutic efficacy in the treatment of cancer. Drugs. 1989;37(2):162-90.

20. Dorr RT. Pharmacology and toxicology of Cremophor EL diluent. The Annals of pharmacotherapy. 1994;28(5 Suppl):S114.

21. Lee M-J, Straubinger RM, Jusko WJ. Physicochemical, Pharmacokinetic and Pharmacodynamic Evaluation of Liposomal Tacrolimus (FK 506) in Rats. Pharmaceutical Research. 1995;12(7): 1055-9.

22. Rowinsky EK, Donehower RC. Paclitaxel (taxol). The New England journal of medicine. 1995;332(15):1004-14.

23. Friedland D, Gorman G, Treat J. Hypersensitivity reactions from taxol and etoposide. Journal of the National Cancer Institute. 1993;85(24):2036.

24. Bhatt P, Lalani R, Mashru R, Misra A. Abstract 2065: Anti-FSHR antibody Fab' fragment conjugated immunoliposomes loaded with cyclodextrin-paclitaxel complex for improved in vitro efficacy on ovarian cancer cells. Cancer research. 2016;76(14 Supplement):2065-.

25. Lam YWF, Chan CYJ, Kuhn JG. Review Pharmacokinetics and pharmacodynamics of the taxanes. Journal of Oncology Pharmacy Practice. 1997;3(2):76-93.

26. Powell JR, Donn KH. The pharmacokinetic basis for $\mathrm{H} 2$-antagonist drug interactions: concepts and implications. Journal of clinical gastroenterology. 1983;5 Suppl 1:95-113.

27. Monsarrat B, Mariel E, Cros S, Garès M, Guénard D, Guéritte-Voegelein F, et al. Taxol metabolism. Isolation and identification of three major metabolites of taxol in rat bile. Drug metabolism and disposition: the biological fate of chemicals. 1990;18(6):895-901.

28. Harris JW, Rahman A, Kim BR, Guengerich FP, Collins JM. Metabolism of taxol by human hepatic microsomes and liver slices: participation of cytochrome P450 3A4 and an unknown P450 enzyme. Cancer research. 1994;54(15):4026-35.

29. Bhatt P, Khatri N, Kumar M, Baradia D, Misra A. Microbeads mediated oral plasmid DNA delivery using polymethacrylate vectors: an effectual groundwork for colorectal cancer. Drug Delivery. 2015;22(6):849-61.

30. Allwood MC, Martin H. The extraction of diethylhexylphthalate (DEHP) from polyvinyl chloride components of intravenous infusion containers and administration sets by paclitaxel injection. International journal of pharmaceutics. 1996;127(1):65-71.

31. Kongshaug M, Cheng LS, Moan J, Rimington C. Interaction of cremophor EL with human plasma. The International journal of biochemistry. 1991;23(4):4738.

32. Liebmann J, Cook JA, Lipschultz C, Teague D, Fisher J, Mitchell JB. The influence of Cremophor EL on the cell cycle effects of paclitaxel (Taxol) in human tumor cell lines. Cancer chemotherapy and pharmacology. 1994;33(4):331-9.

33. Karlowsky JA, Zhanel GG. Concepts on the use of liposomal antimicrobial agents: applications for aminoglycosides. Clinical infectious diseases : an official publication of the Infectious Diseases Society of America. 1992;15(4):654-67.

34. Pinto-Alphandary $\mathrm{H}$, Andremont A, Couvreur P. Targeted delivery of antibiotics using liposomes and 
nanoparticles: research and applications. International journal of antimicrobial agents. 2000;13(3):155-68.

35. Bhatt P, Lalani R, Vhora I, Patil S, Amrutiya J, Misra A, et al. Liposomes encapsulating native and cyclodextrin enclosed paclitaxel: Enhanced loading efficiency and its pharmacokinetic evaluation. International journal of pharmaceutics. 2018;536(1):95-107.

36. Law SL, Hung HY. Properties of acyclovir-containing liposomes for potential ocular delivery. International journal of pharmaceutics. 1998;161(2):253-9.

37. Lasic DD. Sterically Stabilized Vesicles. Angewandte Chemie International Edition in English. 1994;33(17):1685-98.
38. Lalani R, Misra A, Amrutiya J, Patel H, Bhatt P, Patel V. Challenges in Dermal Delivery of Therapeutic Antimicrobial Protein and Peptides. Current Drug Metabolism. 2017;18(5):426-36.

39. $\mathrm{C}^{\smile}$ eh $\mathrm{B}$, Winterhalter $\mathrm{M}$, Frederik PM, Vallner JJ, Lasic DD. Stealth ${ }^{\circledR}$ liposomes: from theory to product. Advanced Drug Delivery Reviews. 1997;24(2):165-77.

40. Lalani RA, Bhatt P, Rathi M, Misra A. Abstract 2063: Improved sensitivity and in vitro efficacy of RGD grafted PEGylated gemcitabine liposomes in RRM1 siRNA pretreated cancer cells. Cancer research. 2016;76(14 Supplement) :2063-. 